\title{
Evaluation of propofol anesthesia in morbidly obese children and adolescents
}

\author{
Vidya Chidambaran ${ }^{1,8^{*}}$, Senthilkumar Sadhasivam ${ }^{1,8}$, Jeroen Diepstraten ${ }^{2}$, Hope Esslinger $^{3}$, Shareen Cox $^{4}$, \\ Beverly M Schnell ${ }^{5}$, Paul Samuels ${ }^{1,8}$, Thomas Inge ${ }^{6,8}$, Alexander A Vinks ${ }^{7,8}$ and Catherijne A Knibbe ${ }^{2,9}$
}

\begin{abstract}
Background: Poor characterization of propofol pharmacokinetics and pharmacodynamics in the morbidly obese (MO) pediatric population poses dosing challenges. This study was conducted to evaluate propofol total intravenous anesthesia (TIVA) in this population.

Methods: After IRB approval, a prospective study was conducted in $20 \mathrm{MO}$ children and adolescents undergoing laparoscopic surgery under clinically titrated propofol TIVA. Propofol doses/infusion rates, hemodynamic variables, times to induction and emergence, and postoperative occurrence of respiratory adverse events (RAE) were recorded, along with intraoperative blinded Bispectral Index/BIS and postoperative Ramsay sedation scores (RSS). Study subjects completed awareness questionnaires on postoperative days 1 and 3. Propofol concentrations were obtained at predetermined intra- and post-operative time points.

Results: Study subjects ranged $9-18$ years (age) and $97-99.9 \%$ (BMI for age percentiles). Average percentage variability of hemodynamic parameters from baseline was $\approx 20 \%$. Patients had consistently below target BIS values (BIS $<40$ for $>90 \%$ of maintenance phase), delayed emergence ( $25.8 \pm 22$ minutes), increased somnolence (RSS $\geq 4$ ) in the first 30 minutes of recovery from anesthesia and $30 \%$ incidence of postoperative RAE, the odds for which increased by $14 \%$ per unit increase in BMI ( $p \leq 0.05$ ). Mean propofol concentration was $6.2 \mathrm{mg} / \mathrm{L}$ during maintenance and $1.8 \mathrm{mg} / \mathrm{L}$ during emergence from anesthesia.
\end{abstract}

Conclusions: Our findings indicate clinical overestimation of propofol requirements and highlight the challenges of clinically titrated propofol TIVA in MO adolescents. In this setting, it may be advantageous to titrate propofol to targeted BIS levels until more accurate weight-appropriate dosing regimens are developed, to minimize relative overdosing and its consequences.

Keywords: Morbidly obese, Bariatric, Propofol, Total intravenous anesthesia, Bispectral index, Anesthetic depth, Pediatric, Adolescents

\section{Background}

Propofol is commonly used for total intravenous anesthesia (TIVA) due to its characteristic ease of titration, rapid onset and offset of action, reduced incidence of postoperative nausea/vomiting [1] and emergence agitation [2]. In the morbidly obese (MO) paediatric population, despite propofol's desirable characteristics, appropriate drug administration is complicated by numerous anatomic and physiological factors that accompany obesity, including

\footnotetext{
* Correspondence: vidya.chidambaran@cchmc.org

'Department of Anesthesia and Paediatrics, Cincinnati Children's Hospital Medical Center, 3333 Burnet Ave, MLC 2001, Cincinnati, OH 45229, USA

${ }^{8}$ University of Cincinnati, Cincinnati, OH, USA

Full list of author information is available at the end of the article
}

increases in total body mass, blood volume, cardiac output and regional blood flow [3]. Inavailability of evidencebased clinical guidelines and an adequate dosing scalar for individualized propofol dosing in MO children and adolescents could adversely impact the quality of TIVA administered to these patients [4].

Recent evidence has highlighted drug dosing issues in obese adults raising concerns at both extremes of drug administration: inadequate anesthesia resulting in intraoperative awareness due to under-dosing propofol [5] and excessive anesthetic administration, resulting in organ hypoperfusion and low processed electroencephalographic

\section{Biomed Central}

(c) 2013 Chidambaran et al.; licensee BioMed Central Ltd. This is an Open Access article distributed under the terms of the Creative Commons Attribution License (http://creativecommons.org/licenses/by/2.0), which permits unrestricted use, distribution, and reproduction in any medium, provided the original work is properly cited. 
index values which could be associated with poor outcomes [6-9]. Although the Bispectral Index/BIS monitor provides quantifiable and continuous assessment of propofol cortical effects in children and adolescents [10-12], it is a common to practice TIVA with propofol in children without BIS monitoring. In this descriptive study in a cohort of MO paediatric patients, we evaluated the effects of propofol TIVA on perioperative outcomes.

\section{Methods}

A prospective study was conducted in $\mathrm{MO}$ children and adolescents between July 2009 and July 2010. The study protocol was approved by Cincinnati Children's Hospital institutional review board and written informed assent / consent was obtained from all participants and/or their guardians as appropriate.

\section{Study subjects}

Inclusion criteria: 1) Males and females between the ages of 5 and 18 years, 2) Body Mass Index (BMI) for age $>95^{\text {th }}$ percentile $\left\{>95^{\text {th }}\right.$ percentile (obese), $>99^{\text {th }}$ percentile (MO) [13]\}; 3) Patients undergoing elective surgery scheduled for a duration of at least 60 minutes.

Exclusion criteria: 1) Severe developmental delay, 2) Known cardiac anomaly, neurological, renal or hepatic disorders, 3) Known allergy to propofol, 4) Skin condition which would preclude placement of BIS sensor on the forehead.

\section{Study protocol}

The patient was brought to the operating room, electrocardiograph, non-invasive blood pressure and pulse oximeter were applied, and an intravenous catheter was established. Before or immediately after induction, an age and head-size appropriate disposable BIS sensor ${ }^{\circ} \mathrm{XP}$, (Aspect Medical Systems, Norwood, MA) was placed on each patient's forehead and connected to the BIS monitor. The BIS monitor screen was covered throughout the procedure to blind the anesthesia personnel to the BIS score and trend screen. Anesthesia was induced with propofol at a standardized infusion rate of $1000 \mu \mathrm{g} \cdot \mathrm{kg}^{-1} \cdot \mathrm{min}^{-1}$ after intravenous injection of lidocaine $30 \mathrm{mg}$. Infusion rates were based on adjusted body weight $(\mathrm{ABW})$ which was calculated using total body weight (TBW) and ideal bodyweight (IBW), as described by Servin et. al. [14], substituting $22 \mathrm{~kg} / \mathrm{m}^{2}$ as Ideal BMI (in Servin's formula) with $50^{\text {th }}$ percentile BMI for age and gender, obtained from Centers for Disease Control and Prevention, National Center for Health Statistics growth charts, United States. (http://www.cdc.gov/growthcharts/. May 30, 2000).

$$
A B W=I B W+0.4^{*}(T B W-I B W)
$$

$$
I B W=I \text { dealBMI }\{\text { Height }(\text { meter })\}^{2}
$$

Patients were asked to count, or called repeatedly in a normal voice until the induction end-point of loss of verbal contact; this was recorded as 'time to induction'. Succinylcholine was administered and the trachea was intubated with an appropriate cuffed endotracheal tube. Anesthesia was maintained with propofol infusion. Vecuronium was titrated to Train-of-Four response (goal: one of four twitches). The induction dose of propofol was followed by propofol infusion at a rate of $250-350 \mu \mathrm{g} / \mathrm{kg} / \mathrm{min}$ for 10 minutes and titrated in $25-50 \mu \mathrm{g} / \mathrm{kg} / \mathrm{min}$ steps (reduced to prevent drop in systolic arterial blood pressure and heart rate below $30 \%$ of baseline values and titrated up when greater than $30 \%$ increase in heart rate or blood pressure occurred in the absence of new painful stimuli). Propofol was infused using calibrated pumps with internal memory and downloading capability. This allowed all real-time rates and rate changes to be recorded, including start and stop time of propofol dosing, propofol infusion rates, and propofol dose adjustments. Fentanyl 50-100 $\mu \mathrm{g}$ was administered after induction and $50 \mu \mathrm{g}$ doses were administered in case of inadequate analgesia (defined as increase in heart rate and/or blood pressure above $30 \%$ of baseline with surgical incision or manipulation). When inadequate anesthesia or analgesia was not considered to be the reason for increase in blood pressure or heart rate, medications to correct hemodynamics were administered. The propofol infusion was decreased by $50 \%$ about 15 minutes before conclusion of surgery and discontinued when skin sutures were being placed. Muscle relaxants were reversed and once the patient was breathing, morphine/hydromorphone was dosed incrementally towards the end of the surgery, titrated to respiratory rate of 14-16 breaths per minute. After clinical confirmation of reversal, the trachea was extubated awake. Patients were then transferred to the recovery area (PACU) and followed until they achieved PACU discharge criteria.

\section{Demographics}

Patient demographics, age, gender, weight (TBW) and height were collected. After computing the BMI, IBW and $\mathrm{ABW}$ were calculated according to equations 1 and 2 . Ideal BMI in Equation 2 is defined as the $50^{\text {th }}$ percentile values from age and sex - specific BMI for age charts at www.cdc.gov. A calculator available at http://www.bcm. $\mathrm{edu} / \mathrm{cnrc} /$ bodycomp/bmiz2.html was used to calculate BMI for age percentiles. Lean body mass (LBM) was calculated using the formula described by Peters et. al. by first estimating Extracellular Volume (ECV) from weight and height [15] according to the following equation.

estimatedLBM $=3.8^{*}$ estimatedECV 


\section{Propofol and opioid doses}

Posthoc calculation of induction dose required to achieve loss of verbal contact was performed by multipying the rate of infusion and time taken to reach the end-point. Means and SD of propofol infusion rates during maintenance were analyzed from pooled data. Propofol maintenance infusion rates were plotted against BIS values and time since start of propofol infusion. Infusion rates of eight patients corresponding to BIS values of 40-60 were then analyzed to derive mean and SD. Hourly opioid use as fentanyl equivalent doses were calculated, based on an equivalence of morphine $10 \mathrm{mg}=2 \mathrm{mg}$ hydromorphone $=$ $100 \mu \mathrm{g}$ fentanyl.

\section{Hemodynamics}

Clinical data including mean, systolic, diastolic blood pressure (MAP, SBP and DBP respectively) and heart rate/HR were recorded electronically every 5 minutes intraoperatively. For each of the measured hemodynamic parameters, percentage difference from baseline (value recorded $5 \mathrm{mi}$ nutes before propofol induction) was calculated according to the following equation.

$$
\% \text { Difference }=100 *\left(\frac{\text { Value-Baseline }}{\text { Baseline }}\right)
$$

\section{BIS}

BIS data were transferred electronically to a computerized record in one-second increments. This included the date and time of BIS data collection, minimum and maximum BIS values, average Signal Quality Index (SQI) and average electromyography (EMG). The smoothing rate of the BIS monitor was set at 15 seconds. Evaluable BIS values were defined as those with Signal Quality Index $>70$.

\section{Blood sampling and propofol analysis}

Blood samples $(1.0 \mathrm{ml})$ were obtained from a dedicated intravenous catheter placed in the upper extremity contralateral to the propofol infusion site. Samples were obtained at baseline prior to the start of propofol, approximately $15,30,45,60,120,180,240$ minutes after the start of the propofol infusion, at 5 and 20 minutes after dose adjustment, just before discontinuation of the propofol infusion and at 5, 10, 15, 30, 45 and $120 \mathrm{mi}-$ nutes after termination of the infusion. Whole-blood samples for propofol analysis were stored at $4^{\circ} \mathrm{C}$ until analysis (within 1 month) by high-performance liquid chromatography with fluorescence detection. The coefficients of variation for the intra-assay and interassay precision over the concentration range from 0.05 to $5.0 \mathrm{mg} . \mathrm{l}^{-1}$ were less than $4.5 \%$ and $7.1 \%$ respectively. The lower limit of quantification was $0.05 \mathrm{mg}^{-1}{ }^{-1}[16]$.

\section{Ramsay sedation scores}

Ramsay Sedation Scores (RSS) were recorded postoperatively about every 10 minutes for the first 30 minutes and thereafter every 30 minutes while in the PACU [17].

\section{Other clinical data}

'Time to eye opening, defined as the time from cessation of propofol infusion to eye opening on verbal command, was noted. Respiratory adverse events (RAE) defined as airway obstruction requiring airway manipulation, episodes of desaturation $(<90 \%)$ and/or need for oxygen for $>120$ minutes in the immediate postoperative period were also recorded. On postoperative day 1 and 3, patients were evaluated using the Structured Awareness Screening Interview created by Davidson et. al. [18].

\section{Statistical analysis}

GraphPad Prism 5 software (GraphPad Software Inc., La Jolla, CA) was used to generate descriptive statistics (mean, standard deviation, median and range for continuous variables and frequencies for categorical variables). Linear, quadratic and cubic trends were tested to detect correlation of weight scalars (TBW, ABW and LBM) with induction dose, in addition to calculation of root mean square errors (MSE) and the regression lines fitted. SAS software () (SAS version 9.2, Cary, North Carolina) was used to perform logistic regression between occurrence of respiratory adverse events and explanatory variables (TBW, IBW, ABW, BMI, propofol amount and duration of propofol infusion) to detect two-tailed $\mathrm{p}$ values with a 95\% Confidence Intervals (CI).

\section{Results}

\section{Demographics}

Patient and surgical characteristics are presented in Table 1. Of 23 patients enrolled, 20 were fully evaluable. One patient withdrew shortly before the procedure (no samples); and two patients were excluded because of difficulty obtaining blood samples from existing intravenous lines. 19 patients met criteria for morbid obesity.

\section{Propofol and opioid doses}

Hourly propofol and fentanyl equivalent doses, as well as calculated induction doses, are presented in Table 2. Data from four patients were excluded from the calculation of propofol induction dose, due to the use of boluses and protocol deviations from standardized infusion for induction. Only linear regression of induction dose and weight scalars was found to be significant. They are depicted in Figure 1. LBM were the most highly correlated to the induction dose with least root MSE. Means and standard deviations (SD) of administered propofol maintenance rates and rates corresponding to BIS 40-60 based on TBW (Figure 2A) and $\mathrm{ABW}$ (Figure 2B) are 
Table 1 Patient characteristics

\begin{tabular}{|c|c|c|c|}
\hline Characteristic & Mean & SD & Range \\
\hline Age (years) & 15.8 & 2.2 & $9-18$ \\
\hline Body weight $(\mathrm{kg})$ & 125.1 & 29.1 & $69.6-184$ \\
\hline Ideal Body Weight $(\mathbf{k g}) \dagger$ & 55.5 & 9.6 & $34.3-74$ \\
\hline Body MASS INDEX/BMI $\left(\mathrm{kg} / \mathrm{m}^{2}\right)$ & 45.6 & 9.2 & $31.3-62.9$ \\
\hline BMI for age percentiles & 99.4 & 0.7 & $97-99.9$ \\
\hline Lean Body Mass $\dagger \dagger$ & 74.6 & 13.6 & 46.7-98.4 \\
\hline Sex $(F-M)$ & \multicolumn{3}{|c|}{$12-8(60 \%-40 \%)$} \\
\hline Co-morbidities ( $n=$ number of patients with condition) & \multicolumn{3}{|c|}{$\begin{array}{l}\text { Insulin Resistance }(n=6) \text { Mild Obstructive Sleep Apnea }(n=7) \text {; Hypertension } \\
(n=5) \text {; Diabetes }(n=1) \text {; Asthma }(n=3) \text {; Dyslipidemia }(n=2) \text {; Gastroesophageal } \\
\text { Reflux }(n=4) \text {; Depression/Anxiety }(n=4) \text {. }\end{array}$} \\
\hline Surgeries ( $n=$ number of patients who underwent the procedure) & \multicolumn{3}{|c|}{$\begin{array}{l}\text { Bariatric - Laparoscopic Gastric bypass or Sleeve Gastrectomy }(n=11) \text {, } \\
\text { Laparoscopic cholecystectomy/appendectomy }(n=6) \text {, Orthopedic procedures on } \\
\text { lower extremities }(n=3) \text {. }\end{array}$} \\
\hline
\end{tabular}

Table 1: Characteristics of the 20 evaluable patients are presented as mean, standard deviation (SD) and range. Derived weight parameters are tldeal Body Weight $=$ Ideal BMI * Height (meter) ${ }^{2}$ where Ideal BMI is defined as the $50^{\text {th }}$ percentile values from age and sex - specific BMI for age charts at www.cdc.gov. IA calculator available at the http://www.bcm.edu/cnrc/bodycomp/bmiz2.html was used to calculate the BMI for age percentiles. ††Calculated using Peters et. al. Formula (see Methods).

presented. Number of paired observations for the latter calculation from data of 8 patients, was 116; evaluable BIS values $0.5-3$ minutes apart were included to maximize available data in that BIS range. Infusion rates administered were consistently higher than those that were found to correlate with BIS 40-60.

\section{Hemodynamics}

Figure 3 shows the mean and SD of the percentage difference from baseline for HR (1A), DBP (1B), SBP (1C) and MAP (1D) from 5 minutes prior to start of propofol to 200 minutes of propofol anesthesia. SBP, MAP and DBP values declined by about $20 \%$, reaching a nadir at about 15 minutes after induction and returning to

Table 2 Propofol dosing and clinical parameters

\begin{tabular}{llll}
\hline Dosing characteristic & Mean & SD & Range \\
\hline Duration of propofol infusion (min) & 135 & 61 & $41-291$ \\
Total amount of propofol (mg) & 3244 & 2205 & $962-10507$ \\
Propofol dose in mg. $\mathrm{kg}^{-1} \mathrm{~h}^{-1}$ & 11.5 & 3 & $6.9-17.8$ \\
Induction dose (mg.kg-1 $\mathrm{TBW})$ & 1.3 & 0.5 & $0.7-2.1$ \\
Induction dose (mg. $\left.\mathrm{kg}^{-1} \mathrm{ABW}\right)$ & 1.9 & 0.8 & $0.9-3.2$ \\
Fentanyl equivalent doses in $\mu \mathrm{g} \cdot \mathrm{h}^{-1}$ & 175 & 70 & $50-300$ \\
Clinical parameters & & & \\
Time to induction (min) & 1.5 & 0.5 & $0.92-2.3$ \\
Time to eye opening (min) & 25.8 & 22.6 & $1.5-93.7$ \\
Incidence of adverse respiratory events & $6 / 20$ (30\%) & \\
Incidence of awareness & $0 / 20$ & & \\
\hline
\end{tabular}

Table 2: Means, standard deviations (SD) and range of propofol and opioid dosing characteristics received by study subjects, and measured clinical outcomes are tabulated. Induction dose is the calculated dose to achieve clinical end point and is expressed per total body weight (TBW) and adjusted body weight (ABW). baseline values in 30-40 minutes. Overall, average percentage variability from baseline was $20 \%$. Labetolol was used in one patient and the data from this patient were excluded from this analysis.

\section{Intraoperative propofol plasma concentrations and BIS scores}

An average of 14 venous samples was collected per patient. In Figure 4A, the means \pm SD of propofol concentrations during different phases of anesthesia are shown. BIS data were not retrievable for one patient due to software malfunction. Figure $4 \mathrm{~B}$ shows the means and SD of BIS values recorded every 5 minutes during the maintenance phase of propofol anesthesia (excluding $1^{\text {st }}$ ten minutes after induction and the last ten minutes of emergence for every patient). It is noteworthy that the BIS values were in the range of $20-40$ for $89.4 \%$ and less than 20 for another $3.9 \%$ of the maintenance phase. Nineteen of twenty patients had BIS levels less than 40 for at least 20 minutes of maintenance.

\section{Postoperative propofol concentrations and RSS}

Propofol concentrations declined to 2.4(1.2) $\mathrm{mg}^{-1} \mathrm{l}^{-1}$ during the first hour of emergence and remained at 1.1(0.6) mg. $\mathrm{l}^{-1}$ during the second hour after the discontinuation of propofol infusion (Figure 4A). Ramsay sedation scores $>4$ were present up to 30 minutes after arrival to the PACU, which indicates deep sedation. Spearman Rank correlation between RSS and the propofol concentrations was found to be 0.65 ( $\mathrm{p}<0.0001)$.

\section{Other clinical data}

Time to induction, eye opening and incidence of awareness are presented in Table 2. Six patients had RAE in 


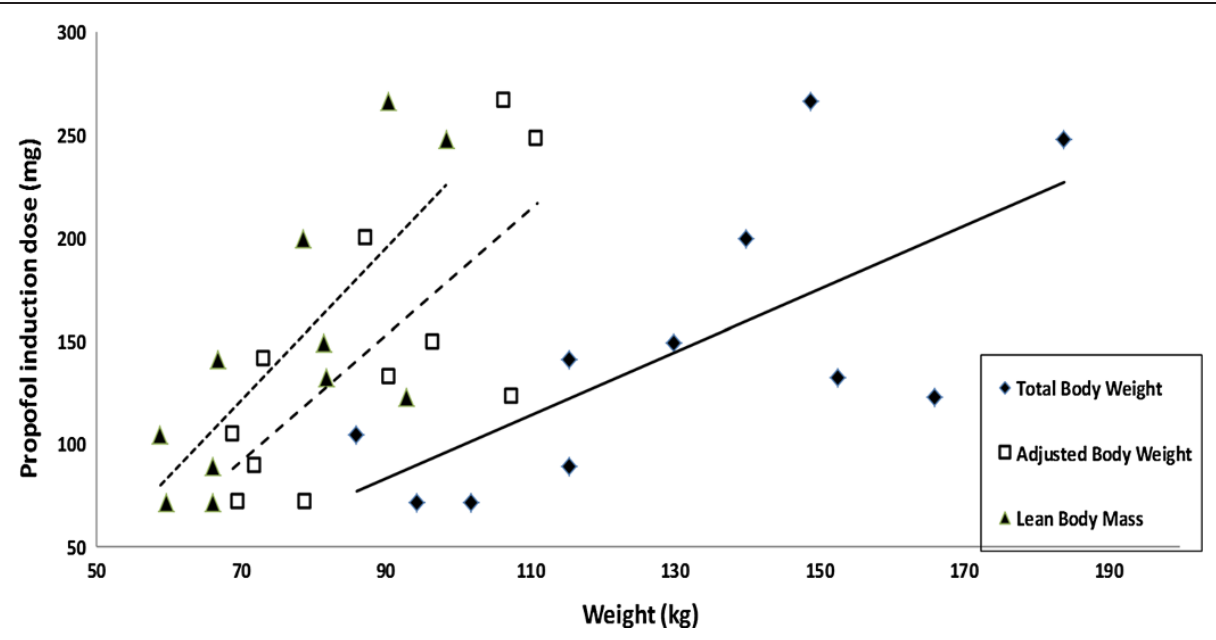

Figure 1 Linear regression of propofol induction dose to weight scalars. Linear regression trendlines for correlation of posthoc calculated induction dose of propofol (titrated to loss of verbal contact) with weight scalars are shown. The correlation coefficients, Root Mean Square Errors (Root MSE) and $p$-values for the correlations were found to be $R^{2}=0.58$, Root MSE $=45.92, p=0.0068$ for Lean Body Mass (LBM), $R^{2}=0.54$, Root $M S E=47.82, p=0.01$ for Adjusted Body Weight (ABW) and $R^{2}=0.5$, Root MSE $=49.61, p=0.0143$ for Total Body Weight (TBW).

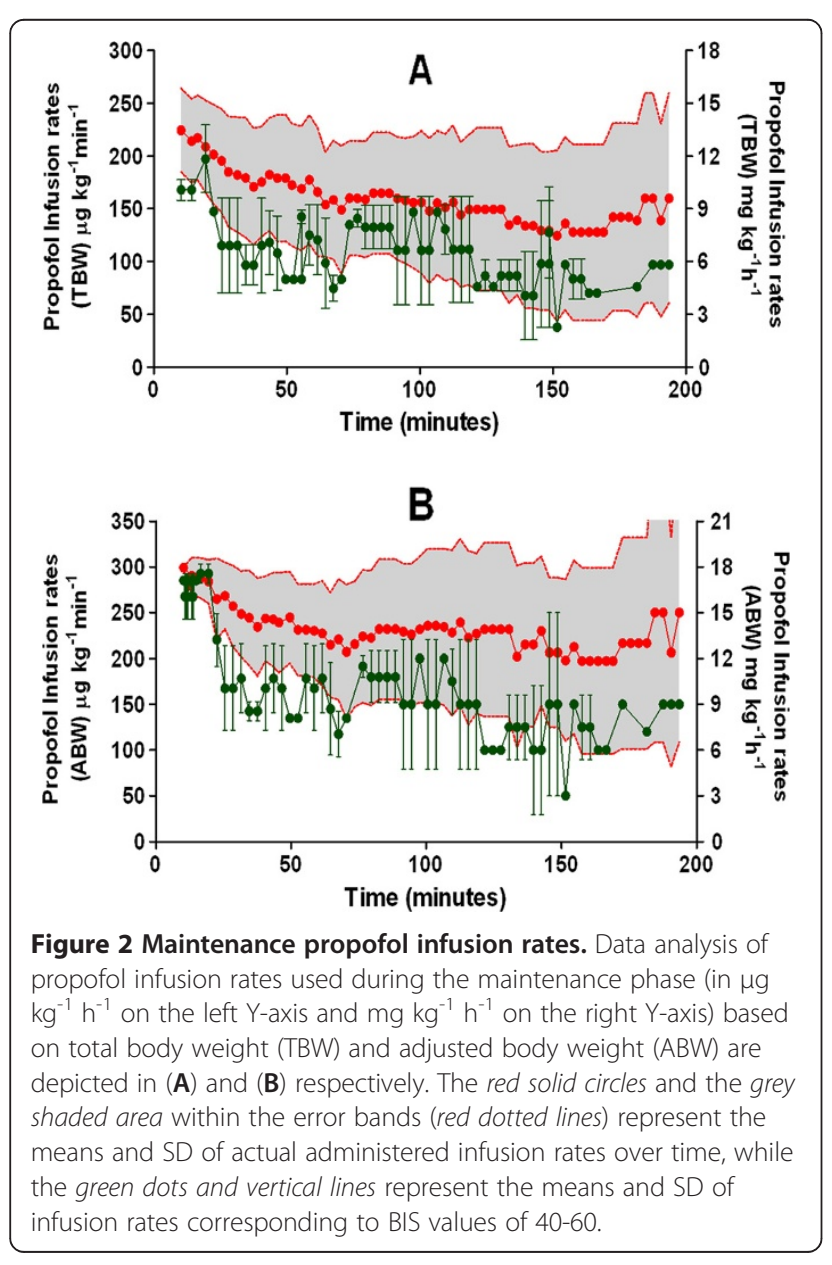

the PACU - one patient had airway obstruction requiring airway manipulation to correct mild hypoxemia, and five others had an extended requirement for oxygen ( $>120$ minutes) to maintain saturation $>90 \%$. BMI was significantly associated with the likelihood of having an adverse respiratory event in the PACU $(p=0.05)$. For every unit increase in BMI, there was a corresponding increase of $14 \%$ in the odds of having an adverse respiratory event.

\section{Discussion}

TIVA with propofol in MO pediatric patients can be challenging in the absence of weight and dosing guidelines.We evaluated the clinical response to propofol anesthesia in this population.

While hemodynamic parameters during propofol TIVA were largely unchanged, BIS values for $\mathrm{MO}$ adolescents were below 40 for $93 \%$ of the maintenance phase. We believe that the increased anesthetic depth was a result of clinical overestimation of propofol requirements. Although our study did not have a BIS control group, our findings that $\mathrm{MO}$ adolescents undergoing clinically titrated propofol TIVA received high propofol doses, is in accordance with what has been reported in obese adults. Gaszynski et. al. demonstrated that obese adults undergoing clinically titrated propofol TIVA without BIS monitoring received higher propofol infusions (10 vs. $5.8 \mathrm{mg} . \mathrm{kg}-1 / \mathrm{h}$ ), consumed more total propofol $(2012 \pm 310 \mathrm{mg}$ vs. $1210 \pm 370 \mathrm{mg})$ and had longer awakening times [19], compared to those who were BIS monitored.

There are two other findings of significance. Firstly, prolonged emergence from anesthesia was observed in our 


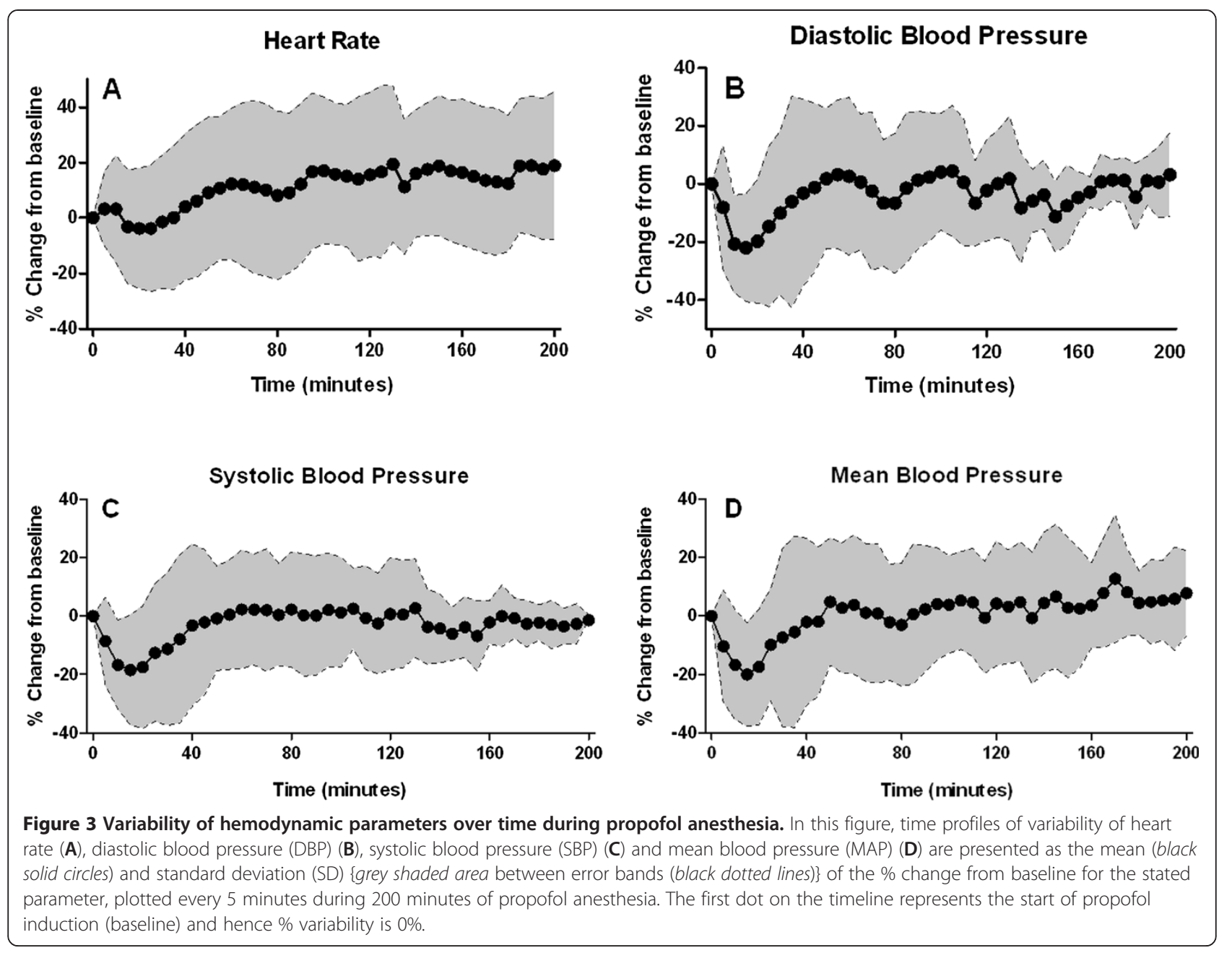

study patients, with an average 'time to eye opening' of $25.9 \pm 22.6 \mathrm{~min}$, compared to $10.3 \pm 5.4$ minutes reported in non-obese children after clinically titrated propofol TIVA $[19,20]$. This was also reflected by deeper levels of sedation (RSS > 4) during the first 30 minutes in the PACU. Although there is some evidence for propofol accumulation and slow washout after continuous propofol infusions in MO adults [21], this has not been supported by clinical data in adults [14]. We believe the prolonged emergence is due to the high propofol doses our study subjects received (mean $=3244 \mathrm{mg}$ or $11.5 \mathrm{mg} \mathrm{kg}^{-1} \mathrm{~h}^{-1}$ ), which positively correlated with the 'time to eye-opening' $(p=0.03)$. Secondly, we note a $30 \%$ incidence of RAE in the immediate postoperative period with a $14 \%$ increased risk of RAE for every unit increase in BMI. Increased risk of RAE after propofol TIVA in obese patients, is supported by Zoremba et. al.'s finding of excessive impairment of pulmonary function in obese adults, two hours after propofol anesthesia [22].

Despite the fact that in clinical settings, propofol is generally administered as a bolus for induction, we chose to use a standardized infusion method for induction. This allowed us to calculate an induction dose based on a clinical endpoint rather than an arbitrary weight-based dose. We noted a high correlation for induction dose to LBM (similar to findings of Ingrande et. al.) [23] and ABW which suggests that the dosing for induction be based on these scalars and not TBW. These findings need to be confirmed with large prospective studies and a formal pharmacokinetic-pharmacodynamic analysis. Pharmacokinetic analysis following this study has been completed and results have been published in an earlier report wherin TBW proved to be the most significant determinant for clearance, while no predictive covariates for volume of distribution were identified [24]. Our infusion regimen was based on ABW as Servin et. al. had used this weight in morbidly obese adults without evidence of propofol accumulation [14]. Our finding that an average infusion rate of $7 \mathrm{mg} \mathrm{kg}^{-1} \mathrm{~h}^{-1}$ TBW during 20-90 minutes of propofol maintenance phase correlates with a BIS of 40-60 (Figure 2A), is higher 


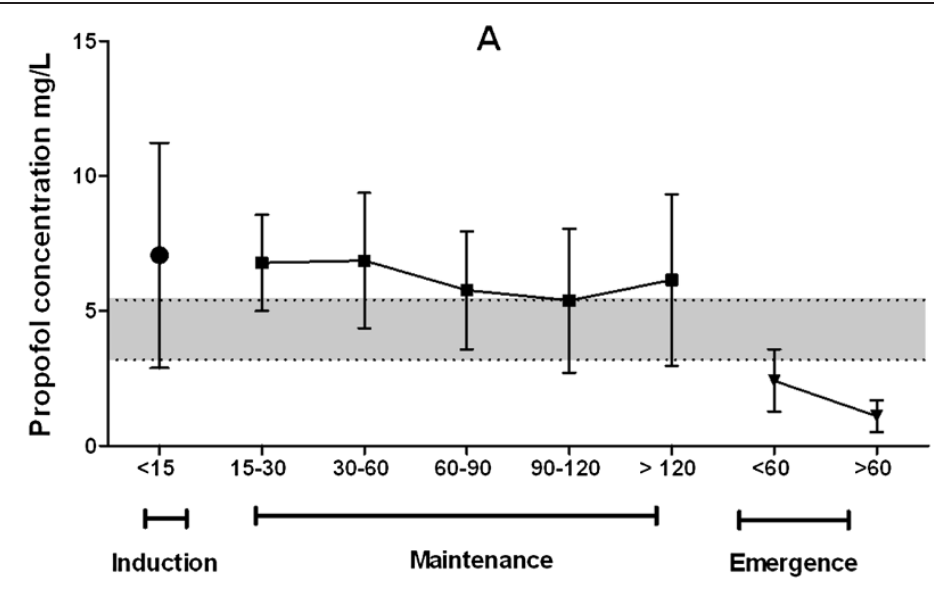

Phases of Anesthesia / Time (minutes)

B

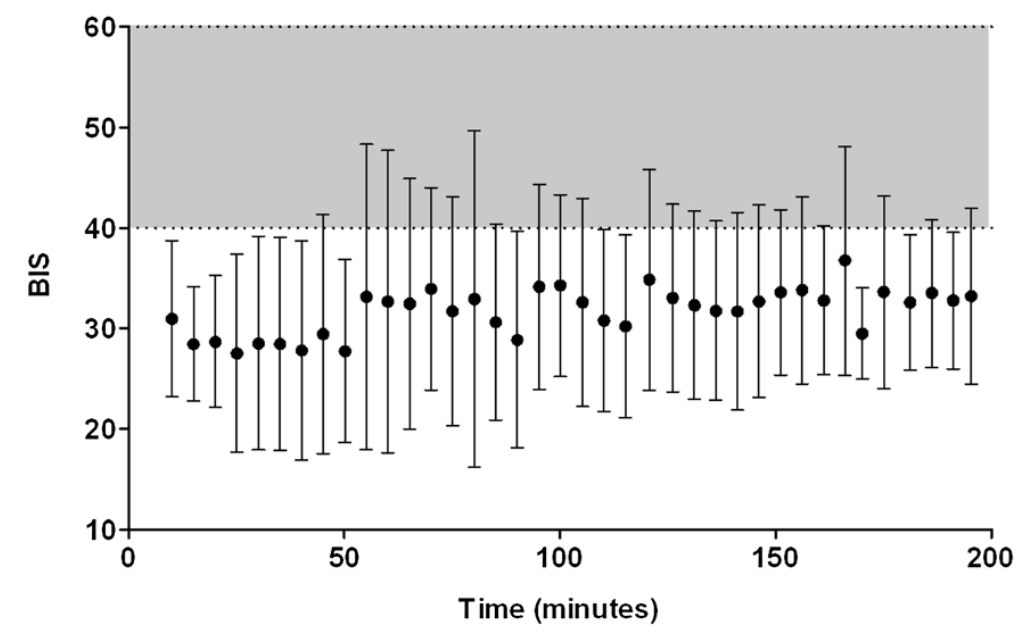

Figure 4 Summary of propofol concentrations during different phases of propofol anesthesia and BIS values over time. (A) represents the means (black solid circles for induction phase, squares for maintenance and inverted triangles for emergence phase) and standard deviations (SD) (black vertical lines) of propofol concentrations during different phases of anesthesia; The propofol concentration during the induction phase (first 15 minutes) was $7.0 \pm 4.1 \mathrm{mg.I^{-1 }}(\mathrm{n}=16)$. The mean (SD)(number of samples) for propofol concentrations collected during 15-30, 30-60, 60-90, 90120 and $>120$ minute time intervals of maintenance anesthesia were 6.8(1.8)(26), 6.9(2.5)(41), 5.8(2.2)(36), 5.4(2.7)(15) and 6.1(3.2)(29) mg..$^{-1}$ respectively. (B) shows the means (black solid circles) and SD (black vertical lines) of blinded BIS values during 10-200 minutes of maintenance phase of propofol anesthesia. Grey shaded areas depict range of propofol concentrations reported to be associated with BIS 50 in children (A) (3.2-5.4 mg.l-1: Riguozzo et. al, 2010) and BIS values generally considered to infer adequate depth of anesthesia (B) (46-60) for this population.

than the recommended rate of 4.6 to $6 \mathrm{mg} \mathrm{kg}^{-1} \mathrm{~h}^{-1} \mathrm{TBW}$ to maintain BIS of 50 in obese adults during the same time period $[14,25]$. Considering that concentrations of $4.3 \pm$ $1.1 \mathrm{mg} . \mathrm{l}^{-1}$ in non-obese children [11] and 3-4 mg. $\mathrm{l}^{-1}$ in obese adults, have been reported to correlate with a BIS of 50 [26], our findings of higher propofol concentrations during maintenance of anesthesia and corresponding lower BIS values suggests that clinical titration of propofol anesthesia in $\mathrm{MO}$ adolescents is not optimal.

Ramsay sedation scores were used to assess sedation in the postoperative period. We used a single non-anesthesia observer to rate RSS in all study subjects to limit interobserver variance. However, caution is required in interpreting correlation of propofol concentrations with RSS as these sedation scores reflect the combination of propofol and opioid effects. We also note that the observational study design allowing clinical titration of propofol doses prevented standardization of dosages. Although dosing of propofol could be affected by differences in opioid doses, it has been reported to not affect the relation between propofol concentrations and BIS [27]. Hence, the lack of standardization of opioid doses would likely not affect our conclusions. Finally, our premise that BIS values below 40 represent very 'deep' anesthesia is debatable, but there is no evidence to the contrary, as none of our patients suffered any awareness. The other dilemma 
concerning the risks associated with excessive anesthesia dosing is still unresolved [28].

\section{Conclusion}

In conclusion, this study presents a detailed descriptive analysis of propofol anesthesia in MO adolescents. Although BIS has been found to improve clinically important outcomes in children undergoing inhalation anesthesia [29], it is not a standard monitor in paediatric anesthetic practice. In MO adults, La Colla et. al. concluded that it is advisable to administer propofol to MO patients by titration to targeted processed-EEG values [30]. Our findings suggest that in the absence of evidence based dosing guidelines for propofol administration in this $\mathrm{MO}$ paediatric population, use of only clinical parameters to dose TIVA with propofol can result in excessive depth of anesthesia. In this setting, BIS monitoring provides anesthesiologists information about real time trend of anesthetic depth and helps prevent excessive propofol administration and associated negative consequences. Our findings also emphasize the need for improved propofol dosing guidelines and monitoring during TIVA in MO adolescents to minimize relative overdosing and its negative consequences.

\section{Abbreviations}

BIS: Bispectral index; MO: Morbidly obese; TIVA: Total Intravenous anesthesia; RSS: Ramsay sedation score.

\section{Competing interests}

The authors declare that they have no competing interests.

\section{Authors' contributions}

VC was involved in design, conduct of the study, analysis of the data, and manuscript preparation, SS helped design, conduct the study, and write the manuscript, JD helped design the study and write the manuscript, HE was involved in conduct of the study, SC helped conduct the study and analyze propofol, BS analyzed the data, PS participated in the conduct of the study and manuscript writing, TI helped conduct of the study, AAV and CAK were involved in designing the study, analysis of the data, and preparation of the manuscript. All authors read and approved the final manuscript.

\section{Acknowledgements}

We would like to acknowledge the Teen Longitudinal Assessment of Bariatric Surgery, Cincinnati Children's Hospital Medical Center (reference www.Teen-LABS.org) for their support; and Aspect Medical Systems, Norwood, MA for loan of BIS monitor for this study. We also acknowledge the help of Elke HJ Krekels, M.Sc. and Lily Ding, PhD. In biostatistics in reviewing the manuscript.

\section{Funding disclosure}

This study was funded by a Translational Research Initiative grant from Cincinnati Children's Research Foundation, Cincinnati Children's Hospital Medical Center, Cincinnati, $\mathrm{OH}$. The findings of this study were presented in part as abstract/poster/oral presentation at the Society of Paediatric Anesthesia Annual meeting at San Antonio, 2011 and American Society of Anesthesiology Annual Meeting, Chicago, 2011.

\section{Author details}

'Department of Anesthesia and Paediatrics, Cincinnati Children's Hospital Medical Center, 3333 Burnet Ave, MLC 2001, Cincinnati, OH 45229, USA. ${ }^{2}$ Division of Pharmacology, Leiden/Amsterdam Center for Drug Research, Leiden, Netherlands. 'Department of Anesthesia, Cincinnati Children's Hospital Medical Center, Cincinnati, OH, USA. ${ }^{4}$ Division of Clinical Pharmacology, Cincinnati Children's Hospital Medical Center, Cincinnati, $\mathrm{OH}$, USA. ${ }^{5}$ Division of Biostatistics and Epidemiology, Cincinnati Children's
Hospital Medical Center, Cincinnati, OH, USA. ${ }^{6}$ Division of Paediatric Surgery, Cincinnati Children's Hospital Medical Center, Cincinnati, OH, USA. ${ }^{7}$ Division of Clinical Pharmacology and Department of Paediatrics, Cincinnati Childrens Hospital Medical Center, Cincinnati, OH, USA. ${ }^{8}$ University of Cincinnati, Cincinnati, OH, USA. 'Department of Clinical Pharmacy, St. Antonius Hospital, Nieuwegein, Netherlands.

Received: 3 August 2012 Accepted: 16 April 2013

Published: 21 April 2013

\section{References}

1. Gurkan Y, Kilickan L, Toker K: Propofol-nitrous oxide versus sevofluranenitrous oxide for strabismus surgery in children. Paediatr Anaesth 1999, 9(6):495-499.

2. Uezono S, Goto T, Terui K, Ichinose F, Ishguro Y, Nakata Y, Morita S: Emergence agitation after sevoflurane versus propofol in pediatric patients. Anesth Analg 2000, 91(3):563-566.

3. Mulla H, Johnson TN: Dosing dilemmas in obese children. Arch Dis Child Educ Pract Ed 2010, 95(4):112-117.

4. Coetzee JF: Total intravenous anaesthesia to obese patients: largely guesswork? Eur J Anaesthesiol 2009, 26(5):359-361.

5. Igarashi T, Nagata O, Iwakiri H, Ikeda M, Uezono S, Ozaki M: [Two cases of intraoperative awareness during intravenous anesthesia with propofol in morbidly obese patients]. Masui 2002, 51(11):1243-1247.

6. Kalkman CJ, Peelen LM, Moons KG: Pick up the pieces: depth of anesthesia and long-term mortality. Anesthesiology 2011, 114(3):485-487.

7. Monk TG, Saini V, Weldon BC, Sigl JC: Anesthetic management and oneyear mortality after noncardiac surgery. Anesth Analg 2005, 100(1):4-10.

8. Kertai MD, Pal N, Palanca BJ, Lin N, Searleman SA, Zhang L, Burnside BA, Finkel KJ, Avidan MS: Association of perioperative risk factors and cumulative duration of low bispectral index with intermediate-term mortality after cardiac surgery in the B-Unaware Trial. Anesthesiology 2010, 112(5):1116-1127.

9. Leslie K, Short TG: Low bispectral index values and death: the unresolved causality dilemma. Anesth Analg 2011, 113(3):660-663.

10. Sadhasivam S, Ganesh A, Robison A, Kaye R, Watcha MF: Validation of the bispectral index monitor for measuring the depth of sedation in children. Anesth Analg 2006, 102(2):383-388.

11. Rigouzzo A, Servin F, Constant I: Pharmacokinetic-pharmacodynamic modeling of propofol in children. Anesthesiology 2010, 113(2):343-352.

12. Jeleazcov C, Ihmsen H, Schmidt J, Ammon C, Schwilden H, Schuttler J, Fechner J: Pharmacodynamic modelling of the bispectral index response to propofol-based anaesthesia during general surgery in children. Br J Anaesth 2008, 100(4):509-516.

13. Barlow SE: Expert committee recommendations regarding the prevention, assessment, and treatment of child and adolescent overweight and obesity: summary report. Pediatrics 2007, 120(Suppl 4):S164-S192.

14. Servin F, Farinotti R, Haberer JP, Desmonts JM: Propofol infusion for maintenance of anesthesia in morbidly obese patients receiving nitrous oxide. A clinical and pharmacokinetic study. Anesthesiology 1993, 78(4):657-665

15. Peters $A M$, Snelling HL, Glass DM, Bird NJ: Estimation of lean body mass in children. Br J Anaesth 2011, 106(5):719-723.

16. Knibbe CA, Koster VS, Deneer VH, Stuurman RM, Kuks PF, Lange R: Determination of propofol in low-volume samples by high-performance liquid chromatography with fluorescence detection. J Chromatogr B: Biomed Sci App/ 1998, 706(2):305-310.

17. Ramsay MA, Savege TM, Simpson BR, Goodwin R: Controlled sedation with alphaxalone-alphadolone. Br Med J 1974, 2(5920):656-659.

18. Davidson AJ, Huang GH, Czarnecki C, Gibson MA, Stewart SA, Jamsen K, Stargatt R: Awareness during anesthesia in children: a prospective cohort study. Anesth Analg 2005, 100(3):653-661. table of contents.

19. Gaszynski T, Strzelczyk J, Machala W, Gaszynski W: Reduction of a total propofol consumption in morbidly obese patients during general anesthesia due to BIS monitoring. Obes Surg 2005, 15(7):1084. author reply 1085.

20. Bhardwaj N, Yaddanapudi S: A randomized trial of propofol consumption and recovery profile with BIS-guided anesthesia compared to standard practice in children. Paediatr Anaesth 2010, 20(9):899-900. 
21. Levitt DG, Schnider TW: Human physiologically based pharmacokinetic model for propofol. BMC Anesthesiol 2005, 5(1):4.

22. Zoremba M, Dette F, Hunecke T, Eberhart L, Braunecker S, Wulf H: A comparison of desflurane versus propofol: the effects on early postoperative lung function in overweight patients. Anesth Analg 2011, 113(1):63-69.

23. Ingrande J, Brodsky JB, Lemmens HJ: Lean body weight scalar for the anesthetic induction dose of propofol in morbidly obese subjects. Anesth Analg 2011, 113(1):57-62.

24. Diepstraten J, Chidambaran V, Sadhasivam S, Esslinger HR, Cox SL, Inge TH Knibbe CA, Vinks AA: Propofol clearance in morbidly obese children and adolescents: influence of age and body size. Clin Pharmacokinet 2012, 51(8):543-551.

25. van Kralingen S, van de Garde EM, van Dongen EP, Diepstraten J, Deneer $\mathrm{VH}$, van Ramshorst B, Knibbe CA: Maintenance of anesthesia in morbidly obese patients using propofol with continuous BIS-monitoring: a comparison of propofol-remifentanil and propofol-epidural anesthesia. Acta Anaesthesiol Belg 2011, 62(2):73-82.

26. Igarashi T, Nagata O, Iwakiri H, Negishi C, Ozaki M: [Reliability of propofol target-controlled infusion in obese patients]. Masui 2009, 58(10):1226-1231.

27. Lysakowski C, Dumont L, Pellegrini M, Clergue F, Tassonyi E: Effects of fentanyl, alfentanil, remifentanil and sufentanil on loss of consciousness and bispectral index during propofol induction of anaesthesia. Br J Anaesth 2001, 86(4):523-527.

28. Lebon G, Warne T, Edwards PC, Bennett K, Langmead CJ, Leslie AG, Tate CG Agonist-bound adenosine A2A receptor structures reveal common features of GPCR activation. Nature 2011, 474(7352):521-525.

29. Messieha ZS, Ananda RC, Hoffman WE, Punwani IC, Koenig HM: Bispectral index system (BIS) monitoring reduces time to extubation and discharge in children requiring oral presedation and general anesthesia for outpatient dental rehabilitation. Pediatr Dent 2005, 27(6):500-504.

30. La Colla L, Albertin A, La Colla G, Ceriani V, Lodi T, Porta A, Aldegheri G, Mangano A, Khairallah I, Fermo I: No adjustment vs. adjustment formula as input weight for propofol target-controlled infusion in morbidly obese patients. Eur J Anaesthesiol 2009, 26(5):362-369.

doi:10.1186/1471-2253-13-8

Cite this article as: Chidambaran et al:: Evaluation of propofol anesthesia in morbidly obese children and adolescents. BMC Anesthesiology 2013 13:8.

\section{Submit your next manuscript to BioMed Central and take full advantage of:}

- Convenient online submission

- Thorough peer review

- No space constraints or color figure charges

- Immediate publication on acceptance

- Inclusion in PubMed, CAS, Scopus and Google Scholar

- Research which is freely available for redistribution 